\title{
Analysis of transcription factors among differentially expressed genes induced by drought stress in Populus davidiana
}

\author{
Bong-Gyu Mun ${ }^{1} \cdot$ Sang-Uk Lee ${ }^{1} \cdot$ Eung-Jun Park ${ }^{2} \cdot$ Hyun-Ho Kim ${ }^{1} \cdot$ \\ Adil Hussain $^{1,3}$ - Qari Muhammad Imran ${ }^{1}$ In-Jung Lee ${ }^{1} \cdot$ Byung-Wook Yun $^{1}$
}

Received: 25 April 2017 / Accepted: 17 June 2017 / Published online: 30 June 2017

(C) The Author(s) 2017. This article is an open access publication

\begin{abstract}
Populus davidiana is native to the Korean Peninsula and is one of the most dominant and abundantly growing forest trees in eastern Asia. Compared to other Populus species such as P. trichocarpa, P. euphratica, and $P$. tremula, relatively little is known about $P$. davidiana. Here, we performed transcriptomic analysis of $P$. davidiana under drought stress induced by $10 \%$ polyethylene glycol. A total of 12,403 and 12,414 differentially expressed genes (DEGs) were successfully annotated with the $P$. trichocarpa reference genome after 6 and $12 \mathrm{~h}$ of treatment, respectively. Of these, a total of 404 genes (238 up-regulated and 166 down-regulated) after $6 \mathrm{~h}$ and 359 genes (187 up-regulated and 172 down-regulated) after $12 \mathrm{~h}$ of treatment were identified as transcription factors. Transcription factors known to be key genes for drought stress response, such as AP2-EREB, WRKY, C2H2, and NAC, were identified. This results suggesting that early induction of these genes affected initiation of transcriptional regulation in response to drought stress. Quantitative real-time PCR results of selected genes showed highly
\end{abstract}

Electronic supplementary material The online version of this article (doi:10.1007/s13205-017-0858-7) contains supplementary material, which is available to authorized users.

Bong-Gyu Mun and Sang-Uk Lee have contributed equally to this work.

Byung-Wook Yun

bwyun@knu.ac.kr

1 School of Applied Biosciences, Kyungpook National University, Daegu 41566, Republic of Korea

2 Division of Forest Biotechnology, Korea Forest Research Institute, Suwon 16631, Republic of Korea

3 Department of Agriculture, Abdul Wali Khan University, Mardan, Pakistan significant $(R=0.93)$ correlation with RNA-Seq data. Interestingly, the expression pattern of some transcription factors was $P$. davidiana specific. The sequence of $P$. davidiana ortholog of $P$. trichocarpa gene POPTR_0018s10230, which plays an important role in plant response to drought, was further analyzed as our RNA-Seq results showed highly significant changes in the expression of this gene following the stress treatment. Sequence of the gene was compared to $P$. trichocarpa gene sequence using cloning-based sequencing. Additionally, we generated a predicted 3D protein structure for the gene product. Results indicated that the amino acid sequence of $P$. davidiana-specific POPTR_0018s10230 is different at six different positions compared to $P$. trichocarpa, resulting in a significantly different structure of the protein. Identifying the transcription factors expressed in $P$. davidiana under drought stress will not only offer clues for understanding the underlying mechanisms involved in drought stress physiology but also serve as a basis for future molecular studies on this species.

Keywords Drought stress - Transcriptome - Poplar · Transcription factors

\section{Introduction}

Populus davidiana is a plant species native to the Korean Peninsula and is one of the most widely distributed forest trees in Korea. The ubiquity of Populus species is indicative of their ability to adapt to diverse environmental conditions, such as cold (Chen et al. 2012), salt (Gu et al. 2004), and drought ( $\mathrm{Li}$ et al. 2011b; Tang et al. 2013b). Drought occurs when there is insufficient irrigation or rainfall for a period, such that soil moisture is reduced to an 
extent that ultimately damages or injures plants. This deficiency is typically accompanied by higher evapotranspiration rates from plant surfaces compared to water absorption by the roots (Jordan and Ritchie 1971).

Drought stress has also been found to be accompanied by other abiotic stresses such as salinity and high temperature stress. Salt and drought stress signal transduction consists of ionic and osmotic homeostasis, detoxification, and growth regulation. The adverse effects of water stress on plant physiology and the mechanisms associated with water stress tolerance and water-use efficiency have been extensively studied (Osakabe et al. 2014). Although Populus trees have a much deeper root system compared to agricultural corps, they are still affected by persistent drought. Persistent drought can influence the structure and growth of roots which in turn negatively affects water uptake leading to the appearance of initial drought symptoms and permanent damage if drought persists (Coder 1999; Kozlowski and Pallardy 2002). Published researches on the molecular mechanisms underpinning responses to drought stress in various crops, such as maize (Avramova et al. 2014), barley (Bedada et al. 2014), potato (Gong et al. 2015), rice (Huang et al. 2014), wheat (Okay et al. 2014), sugarcane (Kido et al. 2012), and soybean (Le et al. 2012), and many other plants, including forest trees such as poplar, pine, and oak (Dong et al. 2014a; Li et al. 2011a). These studies provide useful information regarding the underlying mechanisms and possible management of the problem.

Populus is a promising model of forest trees and/or other woody plants for research on diverse stress responses $(\mathrm{Li}$ et al. 2011a; Qiu et al. 2011; Si et al. 2014; Yan et al. 2012). Moderately drought-stressed Populus euphratica trees have been found to regulate stomatal closure to facilitate higher $\mathrm{CO}_{2}$ accumulation and water absorption for normal growth and development. This is typically accompanied by strong transcriptional regulation of various physiological processes such as stress perception, photoreception, and oxidative stress detoxification at the molecular level (Tang et al. 2013a). Several studies have shown that some species of Populus, such as P. euphratica, are extremely sensitive to drought-induced cavitation (Hukin et al. 2005), whereas P. nigra shows tolerance to drought. Plant cellular responses to various biotic and/or abiotic stresses involve highly complex interconnected networks of signaling pathways, and a systematic understanding of these networks is necessary to comprehend the underlying mechanisms of stress tolerance. An efficient approach for examining the complex internal networks initiated in response to drought stress is discovering genes and metabolic pathways involved in drought stress physiology. This approach may provide clues for the production of drought-tolerant plants (Hamanishi and Campbell 2011).
Recently, high-throughput sequencing technologies have yielded accurate whole-genome sequences on a large scale at low-cost and in a relatively short time. To date, RNA-seq-mediated transcriptome analysis of three Populus species (P. tremula (Paul et al. 2014), P. euphratica (Chen et al. 2014), and $P$. trichocarpa (Kumar et al. 2016) has been reported. More recently, five genes involved in the tolerance to salts, drought, waterlogging, and insect attack have been identified from a transgenic poplar line (Рориlus $\times$ euramericana 'Guariento') based on transcriptome analysis (Zhang et al. 2014).

Transcription factors (TFs) are proteins that regulate the transcription of gene expression by binding to a certain sequence of DNA or/and other protein complex, thereby altering the activity of a protein by either promoting or suppressing its function. Several studies have reported the important role of TFs in response to biotic and abiotic stresses in Populus (Wang et al. 2017). Examples include the NAC and WAKY TFs, as large gene families, involved in plant growth regulation, developmental processes, and stress responses. Although information on the TFs in several Populus species, including $P$. trichocarpa (Hu et al. 2010) and $P$. euphratica (Ma et al. 2016), has been reported, little information is available on the TFs in $P$. davidiana, particularly under drought stress conditions.

Here, we present a transcriptomic analysis of $P$. davidiana and, in particular, the TF families induced by polyethylene glycol (PEG) treatment. PEG is used for induction of high osmotic pressure, thereby causing an effect in vitro similar to drought stress (Muscolo et al. 2014). We anticipate that the findings of this study will serve as a basis for future research on drought stress physiology in Populus species.

\section{Materials and methods}

\section{Plant growth and stress treatment}

P. davidiana seeds were surface-sterilized in $3 \% \mathrm{H}_{2} \mathrm{O}_{2}$, rinsed several times with sterile water (Lendzemo et al. 2009), and then germinated on half-strength Murashige and Skoog (MS) medium (MS $2.2 \mathrm{~g}$, sucrose $10 \mathrm{~g}$, plant agar $8 \mathrm{~g}$; adjusted to $\mathrm{pH}$ 5.8). Four-week-old plants were explanted on MS [4.4 g MS, 3\% sucrose, $0.27 \%$ Gelrite, final $\mathrm{pH} 5.8$; supplemented with $0.5 \mathrm{ppm}$ naphthalene acetic acid (NAA)]. The plants were then incubated for 4 weeks in a controlled environment $\left(22-23{ }^{\circ} \mathrm{C}, 16 \mathrm{~h}\right.$ light and $8 \mathrm{~h}$ dark) for organogenesis. Eight-week-old plants were used for stress treatment. Drought stresses were induced by treatment with $10 \%$ polyethylene glycol (PEG) as described by Kwon et al. (2014). All the treatments were 
performed in triplicates, and samples were collected from analysis at 6 and $12 \mathrm{~h}$ after PEG treatment.

\section{RNA extraction and sequencing}

Total RNA was extracted using Trizol (Invitrogen). Briefly, $P$. davidiana leaf tissues were finely ground in liquid nitrogen using a pestle and mortar and immediately homogenized in $1 \mathrm{ml}$ of Trizol. The homogenized samples were centrifuged $\left(13,000 \mathrm{~g}, 10 \mathrm{~min}, 4{ }^{\circ} \mathrm{C}\right)$, and the supernatant was transferred to fresh tubes. After adding $200 \mu \mathrm{l}$ chloroform, the tubes were vortexed vigorously, kept on ice for $3 \mathrm{~min}$, and then centrifuged. The upper aqueous phase was transferred to fresh tubes containing an equal volume of isopropanol. These tubes were then centrifuged at $4{ }^{\circ} \mathrm{C}$. Precipitated RNA was washed with $75 \%$ ethyl alcohol and re-suspended in RNase-free water.

Sequencing was carried out as described (Hussain et al. 2016). Briefly, RNA libraries were generated using a TruSeq RNA library prep kit v2 (Illumina, USA) and then cDNA was synthesized through fragmentation and hexamer priming of mRNA. Generated cDNA libraries were quantified using a KAPA library quantification kit. A Hiseq-2500 sequencer (Illumina, USA) was used for the RNA-seq.

\section{Measurement of expression levels and identification of differentially expressed genes}

High-quality reads from the raw sequencing reads were matched to the $P$. trichocarpa genome using Ensembl (v.26) (Flicek et al. 2014), and the TopHat program was applied for alignment of RNA-seq reads. Expression levels of genes in the transcriptome were calculated using Cufflinks v2.2.1 (Trapnell et al. 2010) and compared to the $P$. trichocarpa reference data. Additionally, to increase the accuracy of the gene expression level measurements, the data were subjected to multi-read correction and frag-bias corrections. Thereafter, DEGs were identified based on FPKM (fragments per kilo base of transcript per million mapped fragment) and $Q$ value $(<0.05)$ ( $\mathrm{Q}$ value: errorcorrected value after multiple testing). Further, to verify the statistical significance and hierarchical clustering of DEGs, a Heat map was generated using $R$ software (version 3.3.1).

\section{Functional categorization of TFs and comparative analysis}

$P$. davidiana genes were further annotated and analyzed with respect to gene ontology (GO) terms. For this, we referred to the following online databases: NCBI (www. ncbi.nlm.nih.gov), PopGenie (https://popgenie.org), DPTF (https://dptf.cbi.pku.edu.cn), and Plant TFDB (planttfdb_v2.cbi.edu.cn). Similarity BLAST searches were conducted using the NCBI database. GO enrichment analysis was conducted using GO-slim and classified to molecular function, biological process, and cellular location. Transcription factors were analyzed using MapMan (version 3.6.0) to classify functional groups based on a comparison with $P$. trichocarpa as a reference genome from PopGenie and Phytozome (https://phytozome.jgi.doe. gov/). The expression levels of DEGs were validated by qRT-PCR from 10 selected genes, and SNP/indel positions in three genes were compared between $P$. davidiana and $P$. trichocarpa and identified using the Integrative Genomics Viewer (IGV 2.3.72) program.

\section{Structural prediction and protein modeling}

Comparison of the $P$. davidiana sequence with that of $P$. trichocarpa was carried out using the IGV program. The amino acid sequences of selected transcription factors of the two poplar species were submitted to the I-TASSER server for protein model prediction. I-TASSER produced one to five models for each of the poplar sequences. Among these, a model with the highest confidence score (C-score) was selected and analyzed using PyMOL software (LLC, http://www.pymol.org/).

\section{Transcriptomic data analysis and statistical analysis}

To obtain high-quality reads from the raw reads, lowquality reads and adaptors were discarded as described by (Hussain et al. 2016). Briefly, the reads with $>10 \%$ ambiguous bases or with $Q 20<40 \%$ were removed and then quality control was performed using the in-house Theragen software (Theragen Etex, Suwon, Korea). Highquality reads were aligned using TopHat (Trapnell et al. 2009). Expression levels were measured using Cufflinks v2.2.1 (Trapnell et al. 2010). After frag-bias correction and multi-read corrections, differences in the expression of genes were calculated through Cufdiff v2.2.1 (Trapnell et al. 2010) to identify differentially expressed genes with $Q<0.05$. To check correlation between qRT-PCR and RNA-Seq analysis, the log-fold change values of transcripts from both the experiments were plotted in Microsoft Excel 2007 and statistically analyzed to calculate the correlation coefficient $R$.

\section{Results}

\section{Transcriptome analysis}

Figure 1 shows an overall profile of gene expression in response to drought stress induced by $10 \%$ PEG. In order to 


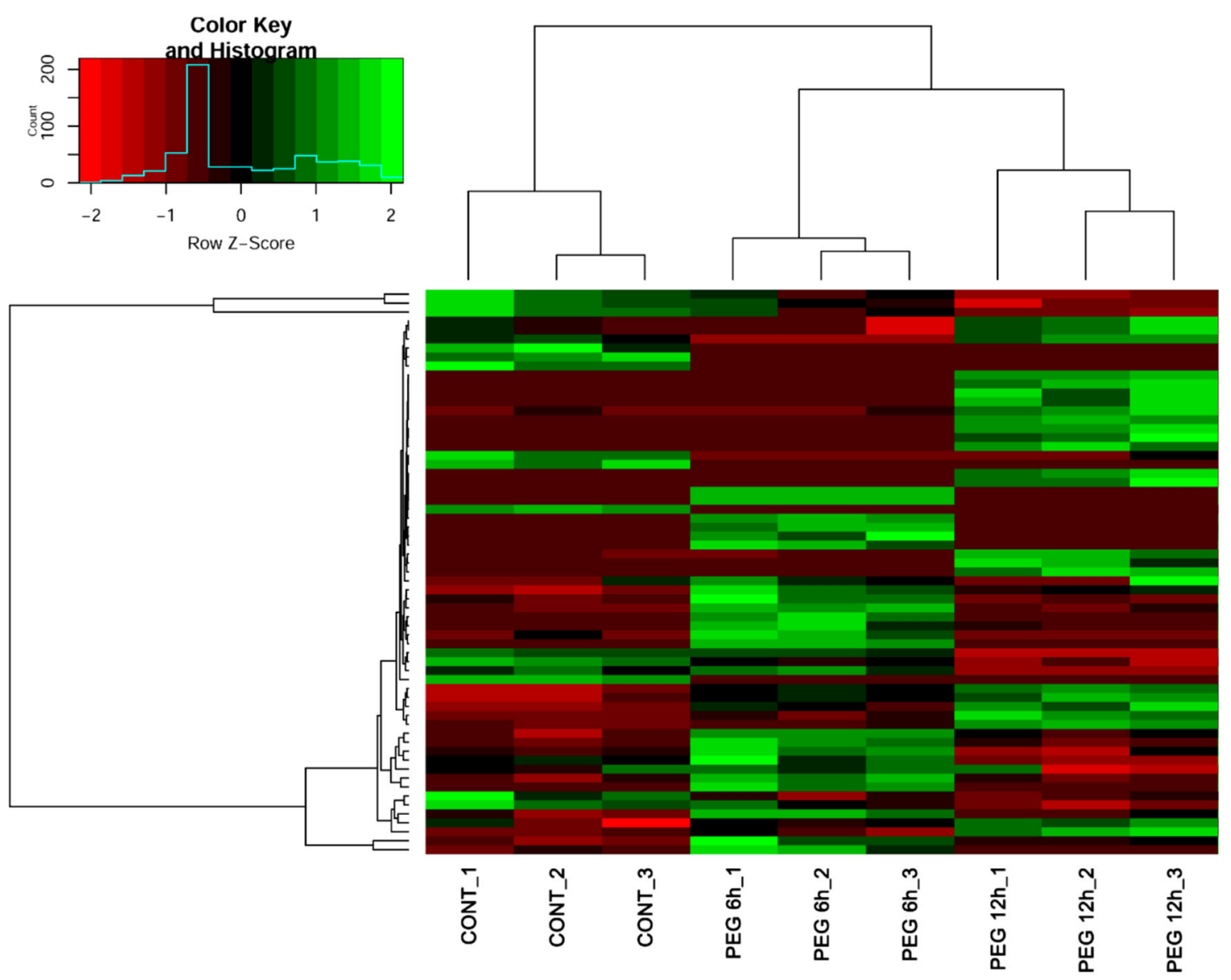

Fig. 1 Heat map of the RNA-Seq-based transcriptome of Populus davidiana after $10 \%$ PEG treatment. The Heat map shows significant differences in the clustering of gene expression in response to $10 \%$

generate the transcriptome data, triplicates of leaf samples were collected for total RNA extraction from un-treated control and $10 \%$ PEG-treated plants grown on MS. These samples were used for cDNA library generation and then sequenced using Illumina high-throughput sequencing. An average of 58.5 million reads were generated from control plants and after 6 and $12 \mathrm{~h}$ of treatment with $10 \%$ PEG by using the genome of $P$. trichocarpa as a reference. The transcriptomic raw sequence data were released to the public through the National Agricultural Biotechnology Information Center (NABIC) database (http://www.nabic. rda.go.kr) with Accession Number (NN-2568-000001).

The transcriptome information for $P$. davidiana is shown in Table 1. A total of 32,087 and 32,330 genes showed differential expression after 6 and $12 \mathrm{~h}$ of treatment with $10 \%$ PEG, respectively. Of these, 12,403 (39\%) and $12,414(39 \%)$, respectively, were successfully mapped and annotated using the $P$. trichocarpa reference from PopGenie and Phytozome. Among these DEGs, a total 404 genes recorded at $6 \mathrm{~h}$ after PEG treatment were identified as transcription factors (238 and 166 genes were up- and polyethylene glycol-induced drought stress in control, $6 \mathrm{~h}$, and $12 \mathrm{~h}$ treatments

down-regulated, respectively), whereas at $12 \mathrm{~h}$ after treatment compared to $6 \mathrm{~h}$, a total of total of 359 DEGs were identified as transcription factors (187 and 172 genes were up- and down-regulated, respectively-Supplementary Figure S1). Significant differences were observed between the two time points with respect to the number, level of expression, and type of responsive genes. In general, the change in transcript accumulation (either up- or downregulated) was greater after $6 \mathrm{~h}$ of stress treatment, indicating that there is a rapid response to water shortage at the molecular level via transcriptional regulation.

\section{Expression pattern of transcription factors}

Our results showed similar transcript expression profiles in various transcription factors at different time points following stress treatment. Detailed analysis of the data revealed that the DEGs contained various TFs families, including WRKY, MYB, bHLH, AP2-AREBP, C2C2-COlike, C2C2-Dof, and $\mathrm{C} 2 \mathrm{H} 2$. In addition, certain unspecified and putative DNA binding domain TFs were identified. 
Table 1 Drought stress-responsive genes of $P$. davidiana compared with $P$. trichocarpa annotation, and the numbers of transcription factors classified using GO terms

\begin{tabular}{|c|c|c|c|c|c|c|}
\hline \multirow{3}{*}{ Group 1} & \multirow[t]{3}{*}{ Group 2} & \multicolumn{5}{|l|}{ Genes } \\
\hline & & \multirow{2}{*}{$\begin{array}{l}\text { Total } \\
\text { differentially } \\
\text { expressed genes }\end{array}$} & \multirow{2}{*}{$\begin{array}{l}\text { Annotation } \\
\text { matched }\end{array}$} & \multirow[t]{2}{*}{ GO term matched } & \multicolumn{2}{|c|}{ Transcription factor } \\
\hline & & & & & Up-regulated & Down-regulated \\
\hline \multirow[t]{2}{*}{ Control } & $6 \mathrm{~h}$ after PEG treatment & 32,087 & $12,403(39 \%)$ & $5252(42 \%)$ & $404(8 \%)$ & \\
\hline & & & & & 238 & 166 \\
\hline \multirow[t]{2}{*}{$6 \mathrm{~h}$ after PEG treatment } & $12 \mathrm{~h}$ after PEG treatment & 32,330 & $12,414(38 \%)$ & $5314(43 \%)$ & $359(7 \%)$ & \\
\hline & & & & & 187 & 172 \\
\hline
\end{tabular}

Interestingly, in a comparison of two time points $(6$ and $12 \mathrm{~h}$ ) after drought induction, most of the genes in the WRKY, MYB, and AP2-EREBP TF families, which play major roles in the response to drought and other abiotic stresses in other Populus species (Campbell 2010; Jiang et al. 2014), were up-regulated at $6 \mathrm{~h}$ but down-regulated at $12 \mathrm{~h}$. However, some genes in the same TF groups (WRKY, MYB, and AP2-EREBP) remained up-regulated even at $12 \mathrm{~h}$ after drought induction. Similar transcript accumulation has observed in AS2, SNF7, and Dicer-like TFs. In contrast, the C2C2-CO-like, G2-like, and TCP TFs were down-regulated at $6 \mathrm{~h}$ but up-regulated at $12 \mathrm{~h}$ (Fig. 2).

\section{Expression pattern of transcription factors in $P$. davidiana}

To investigate the detailed expression pattern of TF families, a pie chart was generated using a total of $404 \mathrm{TFs}$ expressed at $6 \mathrm{~h}$ (238 and 166 genes up- and down-regulated, respectively) and $359 \mathrm{TFs}$ expressed at $12 \mathrm{~h}$ (187 and
172 genes up- and down-regulated) after 10\% PEG treatment (Fig. 3). The results were similar to those obtained using MapMan analysis, with expression of bHLH, MYB, WRKY, $\mathrm{C} 2 \mathrm{H} 2$ and AP2/EREBP domain-containing TFs being commonly up- and down-regulated at both time points. These major TFs accounted for almost half (45\%) of all TFs from the total DEGs in response to PEG-induced drought stress.

Interestingly, bHLH TFs consistently showed up- and down-regulation of transcript levels, regardless of the time point analyzed. This result indicates that bHLH TFs may be involved in multiple signal pathways in adaptation to drought, as reviewed in (Castilhos et al. 2014). A list of the most up- and down-regulated TFs at 6 and $12 \mathrm{~h}$ after drought induction are presented in Supplementary Tables S1 and S2, respectively. In detail, the expression of a drought-responsive transcriptomic element (POPTR_0001s35280) homologous to AS2 domain-containing TFs was highest, being increased by more than 16-fold, followed by that of the PHOR1, WRKY, bHLH, a

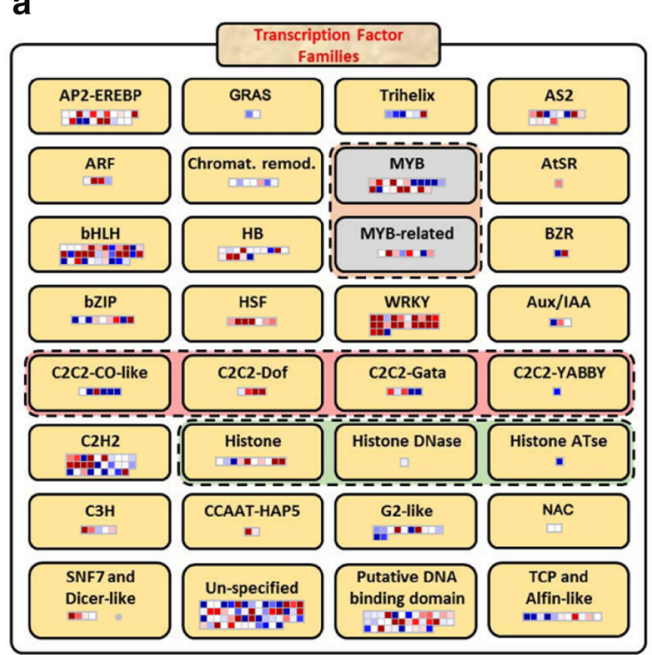

Fig. 2 MapMan analysis of DEGs encoding transcription factors induced in response to drought stress induced by polyethylene glycol (PEG). All transcription factors among the differentially expressed b

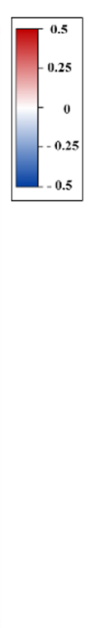

genes were analyzed for functional classification using MapMan. The image shows an overview of the major transcription factors families regulated by $10 \%$ PEG after a $6 \mathrm{~h}$ and b $12 \mathrm{~h}$ of PEG treatment

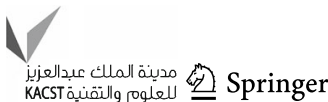


a TFs up-regulated after $6 \mathrm{hrs}$

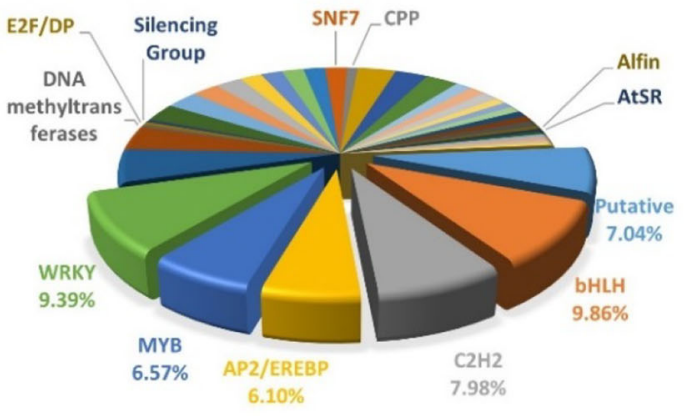

C TFs up-regulated after $12 \mathrm{hrs}$

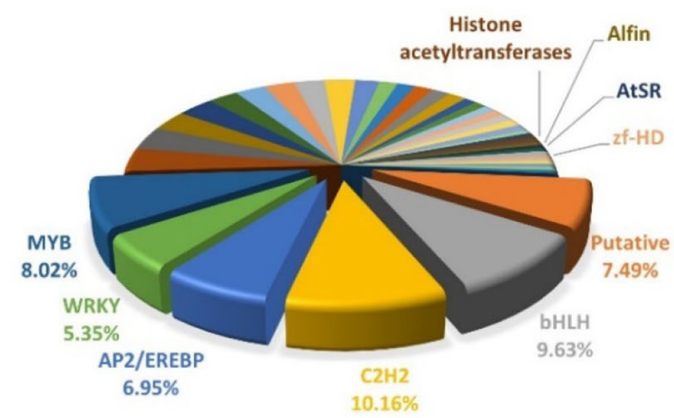

Fig. 3 Expression patterns of various transcription factor (TF) groups in response to osmotic stress. The basic helix-loop-helix (bHLH) TFs were the major group of up-regulated transcripts after $6 \mathrm{~h}$ of polyethylene glycol (PEG) treatment, followed by the WRKY and $\mathrm{C} 2 \mathrm{H} 2$ (a). The putative DNA-binding domain and $\mathrm{C} 2 \mathrm{H} 2$ TFs

AP2/EREBP, and MYB analogs. In contrast, TFs related to C2H2 TFs (POPTR_0010s13400) were down-regulated by more than 8 times, followed by transcripts related to $\mathrm{C} 2 \mathrm{C} 2$ CO-like, ARR, bZIP, and putative DNA binding domain groups of TFs.

After $12 \mathrm{~h}$ of stress treatment, expression of bHLH (POPTR_0015s14070) TFs was increased by more than 32-fold, followed by $\mathrm{C} 2 \mathrm{H} 2$, C2C2(Zn)-GATA, and GARPG2-like TFs. At the same time point, TFs related to the Trihelix (POPTR_0012s11820) group were found to be down-regulated by more than 9.5 times, followed by members of the MADS box, C2H2, AP2-EREBP, and SET-domain-containing groups of TFs. Among the small groups of TFs, Alfin (POPTR_0016s12420) and AtSR (POPTR_0010s15160) were up-regulated after both 6 and $12 \mathrm{~h}$ in response to drought stress treatment. In contrast, S1FA TFs were down-regulated at both time points. In addition, transcript accumulation of SNF7, CPP, DNA methyltransferases, E2F/DP, and silencing TFs was increased at the early time point $(6 \mathrm{~h})$, but decreased as time progressed $(12 \mathrm{~h})$. Although histone acetyltransferases (POPTR_0015s10220) and zf-HD (POPTR_0019s03790) were down-regulated at $6 \mathrm{~h}$, they were up-regulated at

\section{b TFs down-regulated after $6 \mathrm{hrs}$}

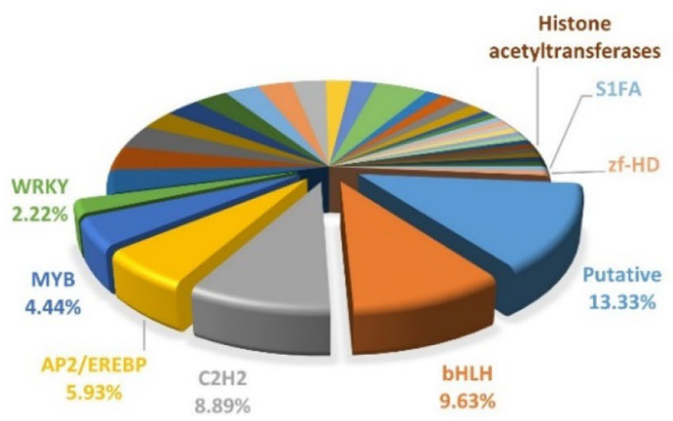

d TFs down-regulated after $12 \mathrm{hrs}$

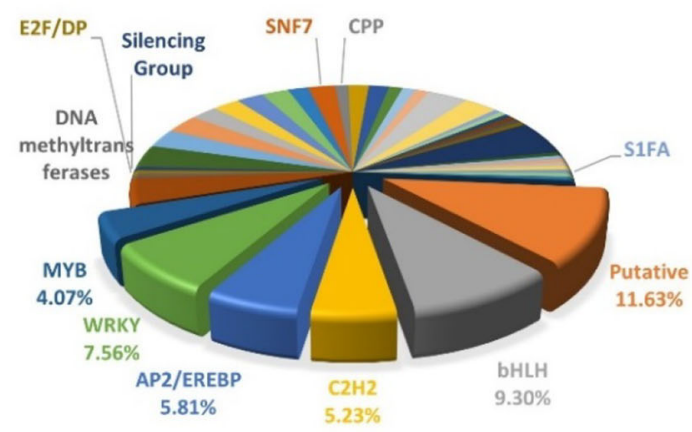

comprised the major group of down-regulated transcripts at $6 \mathrm{~h}$ after PEG treatment (b); however, their expression had increased markedly after $12 \mathrm{~h}$, along with that of $\mathrm{C} 2 \mathrm{H} 2, \mathrm{MYB}$, and AP2/EREBP (c). At this latter time point, the putative DNA-binding domain, bHLH, and WRKY TFs were among the down-regulated TFs (d)

$12 \mathrm{~h}$. Some of these TFs, such as SNF7, CPP, and E2F/DP, have been reported as sub-categories in response to heat stress in Arabidopsis and rice (Ueda et al. 2012). Taken together, these results indicate the diverse roles of these TFs in transcriptional regulation of key genes in drought stress response.

\section{Characterization of transcription factors in $P$. davidiana}

In a comparison of previous studies on other Populus species, TFs such as C2H2, NAC, bHLH, WRKY, and AP2/EREBP were found to be commonly expressed in response to drought stress. In order to determine specific $P$. davidiana $\mathrm{TF}$ characteristics, raw sequences of RNA-seq reads were analyzed by IGV software using the $P$. trichocarpa genome as a reference. For a detailed analysis, three genes (PtrZFP64, PtrZFP99, and PtrZFP103) from C2H2 TFs, which were reported by Liu et al. (2015) to be involved in the drought response in P. trichocarpa and highly up- or down-regulated in the present study, were selected. One of these, PtrZFP103 (POPTR_0018s10230), showed an obvious difference in expression pattern 
between $P$. trichocarpa and $P$. davidiana. According to the study, expression of PtrZFP103 was not altered in P. trichocarpa leaves after drought stress. However, the expression level of this gene was markedly reduced in $P$. davidiana. This result indicates the possibility of sequence differences in certain genes among Populus species and that these differences lead to differential expression of genes in response to stress. To verify differences in gene sequences, transcript isoforms of $P$. davidiana were compared with those of the $P$. trichocarpa genome. We identified 11 sequence differences and confirmed the exact sequence of $P$. davidiana (Supplementary Table 3). This sequence was used in further analyses.

\section{Quantitative real-time PCR validation and SNP confirmation}

To validate PEG-mediated transcriptional changes in the transcriptome, a total of 10 representative genes, encoding transcription factors, i.e., putative DNA binding (POPTR_0013s04170), WRKY (POPTR_0016s10610), homeobox (POPTR_0014s09860), C2H2 (POPTR_0010s13400), Arabidopsis response regulator (POPTR_0014s10160), bHLH (POPTR_0015s14070), and MYB (POPTR_0017s11880) TFs, that showed significant changes in expression levels were selected for qRT-PCR analysis. The sequences of the primers used for qRT-PCR are listed in Supplementary Table 4. We observed significantly similar expression patterns of the transcripts in qRTPCR and RNA-seq data as indicated by the correlation coefficient value $(R=0.93)$ shown in Fig. 4. Additionally, to identify representative $P$. davidiana-specific sequences (SNP), a gene that has been reported to be a drought response gene in P. trichocarpa (Liu et al. 2015) and showed significant changes in our transcriptome data was analyzed using I-Tasser server (Yang et al. 2015). Expression of this gene was substantially decreased after $6 \mathrm{~h}$ of $10 \%$ PEG treatment, and few SNP sequences were detected when compared to P. trichocarpa. Further, to verify the exact sequence of $P$. davidiana, the gene was cloned and sequenced. On the basis of this sequence, a predicted 3D protein structure was generated (Fig. 5). The predicted protein structures of this gene from the two Populus species showed significant differences. Notably, the amino acid sequence was different at six different positions (Fig. 5), resulting in a significantly different conformation of $P$. davidiana-specific protein. The resulting higher change in the expression of this gene following PEG treatment and the significant difference in the sequence/conformation of this protein compared to $P$. trichocarpa further validate its involvement in drought stress regulation.

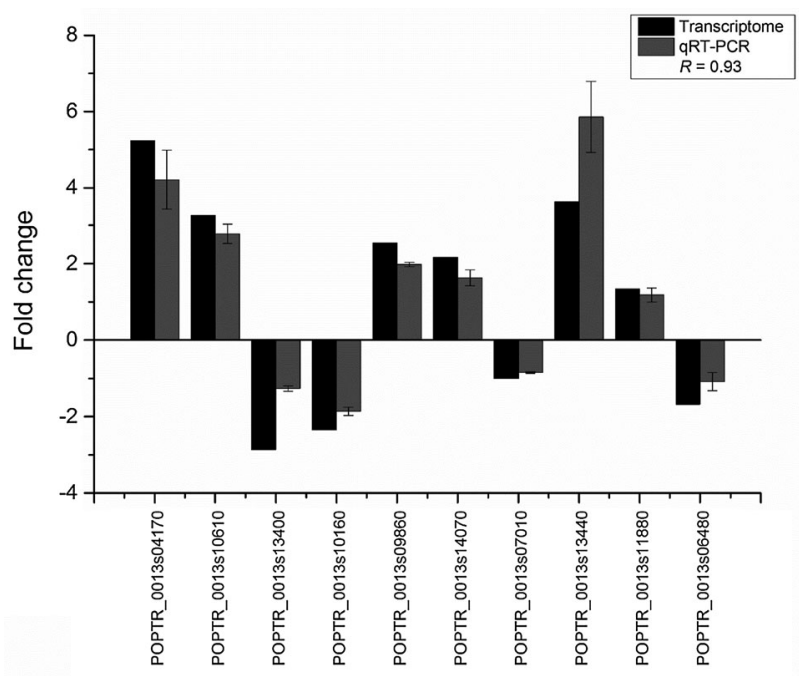

Fig. 4 Quantitative real-time PCR validation of RNA-Seq results for selected genes. Ten differentially expressed genes that showed significant fold changes in their expression levels in transcriptome profiling (black bars) were selected, and their expression levels were validated by qRT-PCR (dark gray). The correlation coefficient $R=0.93$ indicates a significant correlation between the results of qPCR and RNA-Seq

\section{Discussion}

Drought stress is a major problem worldwide because of global climate change. Numerous studies have investigated the drought stress response in crop plant species, and also in a few poplar species. Diverse physiological and biological regulations are involved in the response to drought stress. Among these, TFs, which regulate the activation or deactivation of other genes involved in upstream signal transduction, are good candidates for understanding the underlying mechanisms of the drought response. The role of TFs in abiotic stress responses, including drought stress, has been established in various plant species such as rice and Arabidopsis by using molecular techniques, including microarray analysis and RNA-seq-mediated transcriptome analysis (Fowler and Thomashow 2002; Nakashima et al. 2009; Rabbani et al. 2003; Yamaguchi-Shinozaki and Shinozaki 2006). However, scientific information, particularly that for TFs in the response of perennial forest trees to various abiotic stresses, including drought, is still largely lacking. In the present study, we carried out a transcriptome analysis of $P$. davidiana after application of $10 \%$ PEG to investigate the diverse changes in TFs mediated by drought stress.

In order to determine the total number and types of DEGs, the transcriptomic data were subjected to quality control screening, and DEGs with a cutoff $p$ value $<0.05$ were selected for further studies (Table 1). Of the total DEGs, approximately $40 \%$ were successfully mapped and 


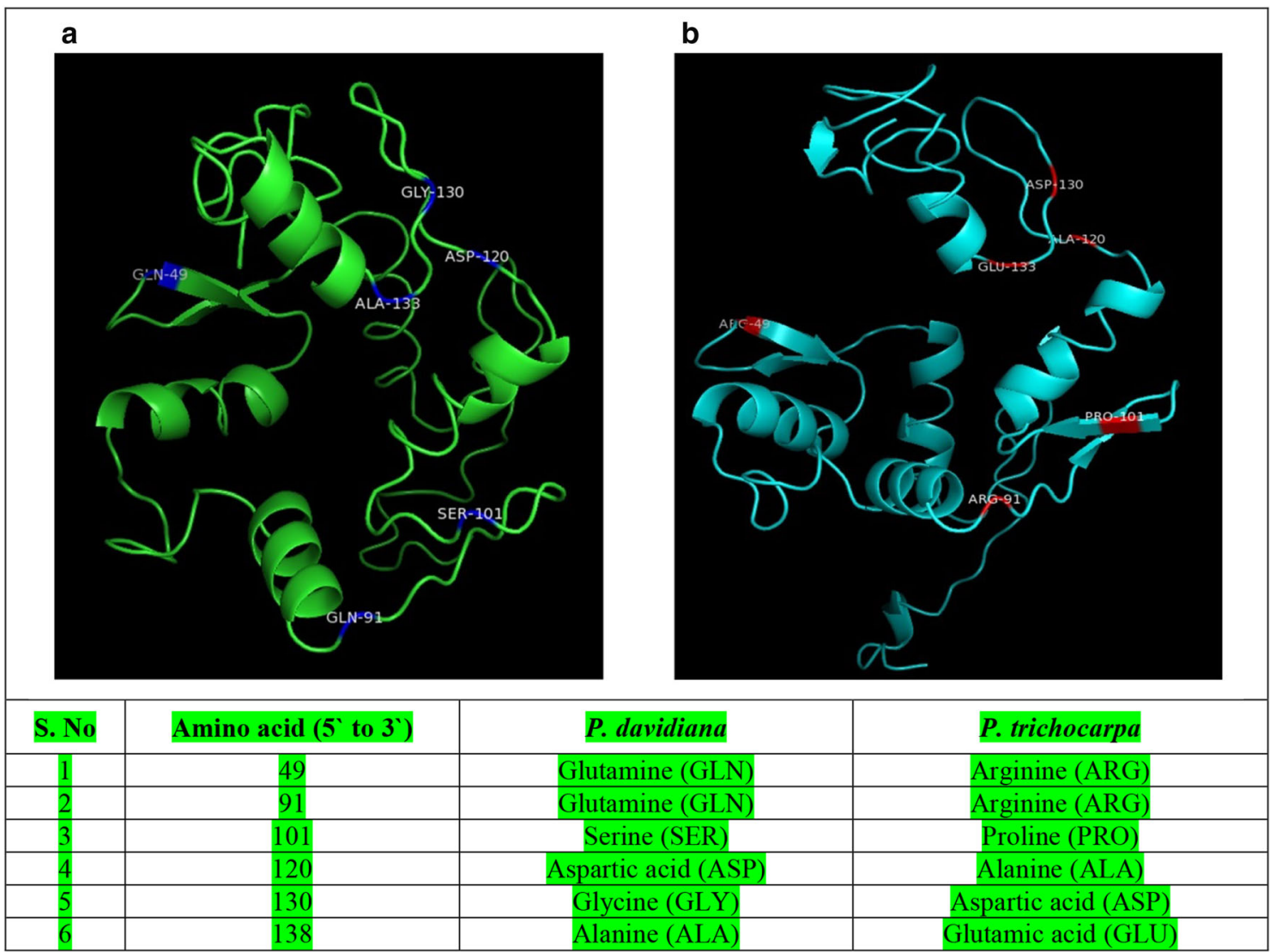

Fig. 5 Comparison of predicted 3D protein structure of POPTR_0018s10230 from P. davidiana (a) and P. trichocarpa (b). The amino acid sequence of POPTR_0018s10230 was analyzed using

annotated using the $P$. trichocarpa reference genome data from PopGenie and Phytozome. A relatively low percentage of TFs was detected in our study compared to that detected for Arabidopsis and rice. This can be attributed to the lack of information on poplar species, for which the functions of many genes have not been predicted. In addition, the numbers of TFs among DEGs were relatively higher at $6 \mathrm{~h}$ compared to $12 \mathrm{~h}$ after PEG treatment. This indicates that plants respond rapidly to drought stress at the molecular level and initiate transcriptional regulation. Once plants detect abiotic stress, such as cold, salt, and drought, diverse of pathways are induced as a means of defense and TFs are among the key factors comprising a regulating defense system. There is a significant overlap between gene expression patterns induced by different types of stresses (Chen et al. 2002; Durrant et al. 2000). TFs families, including WRKY, MYB, bHLH, AP2-AREBP, C2C2-COlike, C2C2-Dof, and $\mathrm{C} 2 \mathrm{H} 2$, well-known TFs that are highly related to the drought responses in Arabidopsis (Saibo et al. 2009), rice (Moumeni et al. 2011) and soybean (Pereira et al. 2011), were most abundantly observed, and these TFs were primarily involved in drought stress response at 6 and $12 \mathrm{~h}$. Among these, WRKY, MYB, and AP2-EREBP TFs, which the I-TASSER server, and the protein structure was generated using PyMOL software. Significant structural changes were attributable to changes in six amino acids

are known to be drought-responsive TFs in other Populus species were up-regulated at $6 \mathrm{~h}$ but down-regulated at $12 \mathrm{~h}$. However, some genes (e.g., POPTR_0013s04170) from the same TF groups remained up-regulated even at $12 \mathrm{~h}$. In contrast, the C2C2-CO-like, G2-like, and TCP TFs were down-regulated at $6 \mathrm{~h}$ but up-regulated at $12 \mathrm{~h}$ (Fig. 2). These observations indicate that there is a significant crosstalk between TFs at the cellular level. For example, after $6 \mathrm{~h}$ of stress treatment, some members of the WRKY and HSF groups of TFs were up-regulated, whereas others were down-regulated. The WRKY signaling cascade is involved in both biotic and abiotic stress responses. Many studies have now established that these genes transcriptionally co-regulate responses to various types of stress. The magnitude of the role of WRKY TFs is demonstrated by the rice OsWRKY13 gene, which regulates the expression of more than 500 stress-responsive genes (Qiu et al. 2008). Overexpression of the PtoWRKY60 gene in Populus tomentosa resulted in a marked increase in the expression of the defense genes PR5.1, PR5.2, PR5.5, and CPR5. Additionally, overexpression of the salicylic acid-inducible PtrWRKY73 from P. trichocarpa enhanced the resistance of Arabidopsis plants to PstDC3000. 
In addition, AP2/EREBP and bHLH TFs groups showed strong differential expression at both the time points examined in the present study. According to the Plant Transcription Factor Database (plntfdb.bio.uni-potsdam.de/ v3.0/), 139 loci have been identified for the AP2/EREBP family of TFs in Arabidopsis, 163 and 138 in Oryza sativa (japonica and indica, respectively), 204 in Zea mays, 338 in Glycine max, and 209 in P. trichocarpa. Similarly, 225 loci have been identified for the bHLH family of TFs in Arabidopsis, 548 in Glycine max, 211 and 169 for Oryza sativa (japonica and indica, respectively), 388 in Zea mays, and 379 in $P$. trichocarpa. These data indicate that AP2/EREBP and bHLH TFs are conserved in the plant kingdom, and previous research has shown that these TFs are part of a gene regulatory network for integrating metabolic, hormonal, sugar, and redox signaling in response to abiotic stresses such as cold and drought (Dietz et al. 2010). For example, the Arabidopsis AP2/EREBP-type TF, AtERF7, plays an important role in ABA response by interacting with protein kinase $\mathrm{PKS} 3$, a global regulator of $\mathrm{ABA}$ responses. AtERF7 binds to the GCC box and acts as a repressor of gene transcription. The stomatal guard cells of Arabidopsis plants overexpressing AtEFR7 show less sensitivity to ABA, and these plants have increased transpiration (Song et al. 2005). Furthermore, one of the bHLH family TFs in Populus, PebHLH35 from Populus euphratica, has been reported as important gene in the drought tolerance response by regulating stomatal development and photosynthesis in Arabidopsis (Dong et al. 2014b).

$P$. davidiana transcriptomic elements homologous to MYB TFs were also a major part of the genes examined in the present study. Functional characterization of $P$. tomentosa R2R3-MYB transcription factor PtoMYB216 revealed its involvement in lignin biosynthesis. A $1.8-\mathrm{kb}$ promoter sequence of this gene fused to the GUS coding sequence and introduced into Arabidopsis showed that GUS expression was restricted to tissues undergoing secondary cell wall formation. Overexpression of this gene enhanced the expression of lignin biosynthetic pathway genes and resulted in lignin deposition, even in cells that are normally unlignified (Tian et al. 2013). In rice, overexpression of the $O s M Y B 48-1$ transcription factor enhanced tolerance to drought and salt stress. OsMYB48-1 was strongly induced by $\mathrm{PEG}, \mathrm{ABA}, \mathrm{H}_{2} \mathrm{O}_{2}$, and dehydration, whereas it was slightly induced by salinity and cold stress. Overexpression of $O s M Y B 48-1$ in rice significantly increases tolerance to drought and salt stress induced by mannitol, $\mathrm{PEG}$, and $\mathrm{NaCl}$, and also regulates the expression of ABA biosynthesis genes (OsNCED4 and OsNCED5) (Xiong et al. 2014). Collectively, the transcriptomic data generated in this study serve as a good platform for further studies investigating and incorporating biotic and abiotic stress tolerance in plants.
TFs related to MYB, AP2-EREBP, C2H2, C2C2-COlike, trihelix, and NAC families are good candidates for plant functional genomic studies. Among these TFs, trihelix and $\mathrm{C} 2 \mathrm{H} 2$ TFs have been reported to be functionally involved in drought response in poplar (Liu et al. 2015; Weng et al. 2012). Interestingly, three genes (POPTR_0016s10460, POPTR_0016s10480, and POPTR_0018s10230) of the C2H2 TF family, reported to be drought response genes in $P$. trichocarpa, were detected in our transcriptome data set containing the 10 most down-regulated and 10 most up-regulated genes. As reported by (Zhang et al. 2015), two species of Populus ( $P$. tremula and $P$. tremuloides) growing in different ecological circumstance showed genetic diversity. In this regard, to identify any genetic difference in $P$. davidiana, the raw RNA-seq reads of three genes were analyzed with IGV software using the $P$. trichocarpa genome as a reference. Among these, POPTR_0018s10230 showed a substantial difference in sequence between $P$. davidiana and $P$. trichocarpa, and the exact sequence of $P$. davidiana was confirmed by clone-based sequencing. The sequence of genes affects the structure of proteins, and such structural changes result in functional changes in proteins (Kishor et al. 2015).

Accordingly, 3D protein structures were generated as shown in Fig. 5. Comparison of the sequences of $P$. davidiana and $P$. trichocarpa revealed a difference in six amino acids. Of these, the proline at position 101 was changed to serine and was associated with a significant structural alteration. Proline is known to function in maintaining structural stability and in abiotic stress response, particularly that to drought and salt stress. For example, proline accumulation was increased fourfold under osmotic stress in a resistant maize cultivar, and proline helps to stabilize subcellular structures in the control of osmolytes. Additionally, exogenous application of proline affects plant growth and photosynthesis, and improves the drought tolerance response (Hayat et al. 2012; Jibran et al. 2015; Rai 2002).

The results of the present study suggest that there is genetic diversity between $P$. davidiana and $P$. trichocarpa and that changes in a few sequences lead to different roles of protein in response to drought stress. On the basis of this information, further biological studies using knock out/down and overexpression mutant plants of these two poplar species would provide further information on the role of this gene in response to drought stress. Taken together, the results of this study of $P$. davidiana TFs under drought stress will expand our knowledge of drought stress-responsive TFs, particularly with respect to how they regulate an intricate system at the cellular level, involving proteins responsible for upstream signal transduction and transcriptional regulation, and also suggest a rich resource

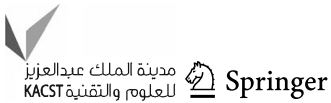


for discovering critical genes related to the drought stress response in $P$. davidiana.

Acknowledgements This work was supported by the Forest Resources Genome Project (Grant Number S111415L070140), Korea Forest Research Institute, Republic of Korea.

\section{Compliance with ethical standards}

Conflict of interest The authors declare that they have no conflict of interest in the publication.

Open Access This article is distributed under the terms of the Creative Commons Attribution 4.0 International License (http:// creativecommons.org/licenses/by/4.0/), which permits unrestricted use, distribution, and reproduction in any medium, provided you give appropriate credit to the original author(s) and the source, provide a link to the Creative Commons license, and indicate if changes were made.

\section{References}

Avramova V, Abdelgawad H, Asard H, Beemster GT (2014) The growth zone of maize leaves subjected to drought stress offers unique possibilities to confirm transcriptome analysis with cellular, physiological and biochemical measurements. Commun Agric Appl Biol Sci 79:111-114

Bedada $\mathrm{G}$ et al (2014) Transcriptome sequencing of two wild barley (Hordeum spontaneum L.) ecotypes differentially adapted to drought stress reveals ecotype-specific transcripts. BMC Genom 15:995. doi:10.1186/1471-2164-15-995

Campbell AS (2010) Drought response of Populus transformed with stress response transcription factors. Master's Thesis, University of Tennessee. http://trace.tennessee.edu/utk_gradthes/690

Castilhos G, Lazzarotto F, Spagnolo-Fonini L, Bodanese-Zanettini MH, Margis-Pinheiro M (2014) Possible roles of basic helixloop-helix transcription factors in adaptation to drought. Plant Sci 223:1-7. doi:10.1016/j.plantsci.2014.02.010

Chen WQ et al (2002) Expression profile matrix of Arabidopsis transcription factor genes suggests their putative functions in response to environmental stresses. Plant Cell 14:559-574. doi: $10.1105 /$ tpc. 010410

Chen L, Zhang YY, Ren YY, Xu JC, Zhang ZY, Wang YW (2012) Genome-wide identification of cold-responsive and new microRNAs in Populus tomentosa by high-throughput sequencing. Biochem Biophy Res Commun 417:892-896. doi:10.1016/j. bbrc.2011.12.070

Chen J, Tian Q, Pang T, Jiang L, Wu R, Xia X, Yin W (2014) Deepsequencing transcriptome analysis of low temperature perception in a desert tree Populus euphratica. BMC Genom 15:326. doi:10.1186/1471-2164-15-326

Coder KD (1999) Drought damage to trees. University of Georgia Daniel B. Warnell School of Forest Resources Extension publication. http://www.forestry.uga.edu/efr

Dietz KJ, Vogel MO, Viehhauser A (2010) AP2/EREBP transcription factors are part of gene regulatory networks and integrate metabolic, hormonal and environmental signals in stress acclimation and retrograde signalling. Protoplasma 245:3-14. doi:10. 1007/s00709-010-0142-8

Dong Y, Wang C, Han X, Tang S, Liu S, Xia X, Yin W (2014a) A novel bHLH transcription factor PebHLH35 from Populus euphratica confers drought tolerance through regulating stomatal development, photosynthesis and growth in Arabidopsis.
Biochem Biophys Res Commun 450:453-458. doi:10.1016/j. bbrc.2014.05.139

Dong Y, Wang CP, Han X, Tang S, Liu S, Xia XL, Yin WL (2014b) A novel bHLH transcription factor PebHLH35 from Populus euphratica confers drought tolerance through regulating stomatal development, photosynthesis and growth in Arabidopsis. Biochem Bioph Res Commun 450:453-458. doi:10.1016/j.bbrc. 2014.05.139

Durrant WE, Rowland O, Piedras P, Hammond-Kosack KE, Jones JDG (2000) cDNA-AFLP reveals a striking overlap in racespecific resistance and wound response gene expression profiles. Plant Cell 12:963-977. doi:10.1105/tpc.12.6.963

Flicek P et al (2014) Ensembl 2014. Nucleic Acids Res 42:D749_ D755. doi:10.1093/nar/gkt1196

Fowler S, Thomashow MF (2002) Arabidopsis transcriptome profiling indicates that multiple regulatory pathways are activated during cold acclimation in addition to the CBF cold response pathway. Plant Cell 14:1675-1690. doi:10.1105/tpc.003483

Gong L et al (2015) Transcriptome profiling of the potato (Solanum tuberosum L.) plant under drought stress and water-stimulus conditions. PLoS One 10:e0128041. doi:10.1371/journal.pone. 0128041

Gu RS, Fonseca S, Puskas LG, Hackler L, Zvara A, Dudits D, Pais MS (2004) Transcript identification and profiling during salt stress and recovery of Populus euphratica. Tree Physiol 24:265-276

Hamanishi ET, Campbell MM (2011) Genome-wide responses to drought in forest trees. Forestry 84:273-283. doi:10.1093/ forestry/cpr012

Hayat S, Hayat Q, Alyemeni MN, Wani AS, Pichtel J, Ahmad A (2012) Role of proline under changing environments: a review. Role of proline under changing environments: a review Plant Signal Behav 7:1456-1466. doi:10.4161/psb.21949

Hu RB, Qi GA, Kong YZ, Kong DJ, Gao QA, Zhou GK (2010) Comprehensive analysis of NAC domain transcription factor gene family in Populus trichocarpa. BMC Plant Biol 10:145. doi:10.1186/1471-2229-10-145 (Artn)

Huang L, Zhang F, Zhang F, Wang W, Zhou Y, Fu B, Li Z (2014) Comparative transcriptome sequencing of tolerant rice introgression line and its parents in response to drought stress. BMC Genom 15:1026. doi:10.1186/1471-2164-15-1026

Hukin D, Cochard H, Dreyer E, Le Thiec D, Bogeat-Triboulot MB (2005) Cavitation vulnerability in roots and shoots: does Populus euphratica Oliv., a poplar from arid areas of Central Asia, differ from other poplar species? J Exp Bot 56:2003-2010. doi:10. 1093/jxb/eri198

Hussain A et al (2016) Nitric oxide mediated transcriptome profiling reveals activation of multiple regulatory pathways in Arabidopsis thaliana. Front Plant Sci. doi:10.3389/Fpls.2016.00975 (Artn 975)

Jiang YZ et al (2014) Genome-wide identification and characterization of the Populus WRKY transcription factor family and analysis of their expression in response to biotic and abiotic stresses. J Exp Bot 65:6629-6644. doi:10.1093/jxb/eru381

Jibran R et al (2015) Staying green postharvest: how three mutations in the Arabidopsis chlorophyll b reductase gene NYC1 delay degreening by distinct mechanisms. J Exp Bot 66:6849-6862. doi:10.1093/jxb/erv390

Jordan WR, Ritchie JT (1971) Influence of soil water stress on evaporation, root absorption, and internal water status of cotton. Plant Physiol 48:783-788

Kido EA et al (2012) New insights in the sugarcane transcriptome responding to drought stress as revealed by superSAGE. TheScientificWorldJournal 2012:821062. doi:10.1100/2012/ 821062

Kishor PBK, Kumari PH, Sunita MSL, Sreenivasulu N (2015) Role of proline in cell wall synthesis and plant development and its 
implications in plant ontogeny. Front Plant Sci 6 doi: 10.3389/ fpls.2015.00544 (ARTN 544)

Kozlowski TT, Pallardy SG (2002) Acclimation and adaptive responses of woody plants to environmental stresses. Bot Rev 68:270-334 8101(2002)068[0270:Aaarow]2.0.Co;2

Kumar S, Kalra S, Singh B, Kumar A, Kaur J, Singh K (2016) RNASeq mediated root transcriptome analysis of Chlorophytum borivilianum for identification of genes involved in saponin biosynthesis. Funct Integr Genom 16:37-55. doi:10.1007/ s10142-015-0465-9

Kwon S-H, Kwon H-K, Kim W, Noh EW, Kwon M, Choi YI (2014) Identification of salt and drought inducible glutathione S-transferase genes of hybrid poplar. J Plant Biotechnol 41:26-32

Le DT et al (2012) Differential gene expression in soybean leaf tissues at late developmental stages under drought stress revealed by genome-wide transcriptome analysis. PloS One 7:e49522. doi:10.1371/journal.pone.0049522

Lendzemo V et al (2009) The arbuscular mycorrhizal host status of plants can not be linked with the Striga seed-germinationactivity of plant root exudates. J Plant Dis Prot 116:86-89

Li B, Qin Y, Duan H, Yin W, Xia X (2011a) Genome-wide characterization of new and drought stress responsive microRNAs in Populus euphratica. J Exp Bot 62:3765-3779. doi:10. 1093/jxb/err051

Li BS, Qin YR, Duan H, Yin WL, Xia XL (2011b) Genome-wide characterization of new and drought stress responsive microRNAs in Populus euphratica. J Exp Bot 62:3765-3779. doi:10. 1093/jxb/err051

Liu QG, Wang ZC, Xu XM, Zhang HZ, Li CH (2015) Genome-wide analysis of $\mathrm{C} 2 \mathrm{H} 2$ Zinc-finger family transcription factors and their responses to abiotic stresses in poplar (Populus trichocarpa). PLoS One 10:e0134753. doi:10.1371/journal.pone. 0134753 (ARTN)

Ma XD, Ma JC, Fan D, Li CF, Jiang YZ, Luo KM (2016) Genomewide identification of TCP family transcription factors from Populus euphratica and their involvement in leaf shape regulation. Sci Rep Uk 6. doi: 10.1038/srep32795 (ARTN 32795)

Moumeni A et al (2011) Comparative analysis of root transcriptome profiles of two pairs of drought-tolerant and susceptible rice near-isogenic lines under different drought stress. BMC Plant Biol 11:174. doi:10.1186/1471-2229-11-174 (Artn)

Muscolo A, Sidari M, Anastasi U, Santonoceto C, Maggio A (2014) Effect of PEG-induced drought stress on seed germination of four lentil genotypes J Plant Interact 9:354-363. doi:10.1080/ 17429145.2013 .835880

Nakashima K, Ito Y, Yamaguchi-Shinozaki K (2009) Transcriptional regulatory networks in response to abiotic stresses in Arabidopsis and grasses. Plant Physiol 149:88-95. doi:10.1104/pp.108. 129791

Okay S, Derelli E, Unver T (2014) Transcriptome-wide identification of bread wheat WRKY transcription factors in response to drought stress. Mol Genet Genom MGG 289:765-781. doi:10. 1007/s00438-014-0849-x

Osakabe Y, Osakabe K, Shinozaki K, Tran LSP (2014) Response of plants to water stress. Front Plant Sci 5:86. doi:10.3389/Fpls. 2014.00086 (Artn)

Paul A, Jha A, Bhardwaj S, Singh S, Shankar R, Kumar S (2014) RNA-seq-mediated transcriptome analysis of actively growing and winter dormant shoots identifies non-deciduous habit of evergreen tree tea during winters. Sci Rep 4:5932. doi:10.1038/ srep05932

Pereira SS et al (2011) Transcription factors expressed in soybean roots under drought stress. Genet Mol Res 10:3689-3701. doi:10.4238/2011.October.21.5
Qiu DY, Xiao J, Xie WB, Liu HB, Li XH, Xiong LZ, Wang SP (2008) Rice gene network inferred from expression profiling of plants overexpressing $O s W R K Y 13$, a positive regulator of disease resistance. Mol Plant 1:538-551. doi:10.1093/mp/ssn012

Qiu Q et al (2011) Genome-scale transcriptome analysis of the desert poplar Populus euphratica. Tree Physiol 31:452-461. doi:10. 1093/treephys/tpr015

Rabbani MA et al (2003) Monitoring expression profiles of rice genes under cold, drought, and high-salinity stresses and abscisic acid application using cDNA microarray and RNA get-blot analyses. Plant Physiol 133:1755-1767. doi:10.1104/pp.103.025742

Rai VK (2002) Role of amino acids in plant responses to stresses. Biol Plant 45:481-487. doi:10.1023/A:1022308229759

Saibo NJM, Lourenco T, Oliveira MM (2009) Transcription factors and regulation of photosynthetic and related metabolism under environmental stresses. Ann Bot Lond 103:609-623. doi:10. 1093/aob/men227

Si J, Zhou T, Bo W, Xu F, Wu R (2014) Genome-wide analysis of salt-responsive and novel microRNAs in Populus euphratica by deep sequencing. BMC Genet 15(Suppl 1):S6. doi:10.1186/ 1471-2156-15-S1-S6

Song CP, Agarwal M, Ohta M, Guo Y, Halfter U, Wang P, Zhu JK (2005) Role of an Arabidopsis AP2/EREBP-type transcriptional repressor in abscisic acid and drought stress responses. Plant Cell 17:2384-2396. doi:10.1105/tpc.105.033043

Tang S et al (2013a) Populus euphratica: the transcriptomic response to drought stress. Plant Mol Biol 83:539-557. doi:10.1007/ s11103-013-0107-3

Tang S et al (2013b) Populus euphratica: the transcriptomic response to drought stress. Plant Mol Biol 83:539-557. doi:10.1007/ s11103-013-0107-3

Tian Q, Wang X, Li C, Lu W, Yang L, Jiang Y, Luo K (2013) Functional characterization of the poplar $R 2 R 3-M Y B$ transcription factor PtoMYB216 involved in the regulation of lignin biosynthesis during wood formation. PLoS One 8:e76369. doi:10.1371/journal.pone.0076369

Trapnell C, Pachter L, Salzberg SL (2009) TopHat: discovering splice junctions with RNA-Seq. Bioinformatics 25:1105-1111. doi:10. 1093/bioinformatics/btp120

Trapnell $\mathrm{C}$ et al (2010) Transcript assembly and quantification by RNA-Seq reveals unannotated transcripts and isoform switching during cell differentiation. Nat Biotechnol 28:511-515. doi:10. $1038 /$ nbt. 1621

Ueda K, Matsuura H, Yamaguchi M, Demura T, Kato K (2012) Genome-wide analyses of changes in translation state caused by elevated temperature in Oryza sativa. Plant Cell Physiol 53:1481-1491. doi:10.1093/pcp/pcs092

Wang H, Zhao S, Gao Y, Yang J (2017) Characterization of Dof transcription factors and their responses to osmotic stress in poplar (Populus trichocarpa). PLoS One 12:e0170210

Weng H, Yoo CY, Gosney MJ, Hasegawa PM, Mickelbart MV (2012) Poplar GTL1 Is a Ca2 +/calmodulin-binding transcription factor that functions in plant water use efficiency and drought tolerance. PLoS One 7:e32925. doi:10.1371/journal.pone. 0032925 (ARTN)

Xiong $\mathrm{H}$ et al (2014) Overexpression of OsMYB48-1, a novel MYBrelated transcription factor, enhances drought and salinity tolerance in rice. PLoS One 9:e92913. doi:10.1371/journal. pone. 0092913

Yamaguchi-Shinozaki K, Shinozaki K (2006) Transcriptional regulatory networks in cellular responses and tolerance to dehydration and cold stresses. Annu Rev Plant Biol 57:781-803. doi:10. 1146/annurev.arplant.57.032905.105444

Yan DH, Fenning T, Tang S, Xia X, Yin W (2012) Genome-wide transcriptional response of Populus euphratica to long-term

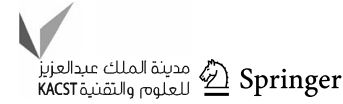


drought stress. Plant Sci 195:24-35. doi:10.1016/j.plantsci.2012. 06.005

Yang JY, Yan RX, Roy A, Xu D, Poisson J, Zhang Y (2015) The I-TASSER Suite: protein structure and function prediction. Nat Methods 12:7-8. doi:10.1038/nmeth.3213

Zhang W et al (2014) Transcriptome sequencing of transgenic poplar (Populus $\times$ euramericana 'Guariento') expressing multiple resistance genes. BMC Genet 15(Suppl 1):S7. doi:10.1186/ 1471-2156-15-S1-S7

Zhang $\mathrm{C}$ et al. (2015) Genetic diversity in aspen and its relation to arthropod abundance. Front Plant Sci 5. doi: 10.3389/fpls.2014. 00806 (ARTN 806) 